\title{
A Unique Common Fixed Point Theorem in Cone Metric Type Spaces
}

\author{
K. P. R. Rao ${ }^{1}$, G.N.V.Kishore ${ }^{2, *}$, P.R.Sobhana Babu ${ }^{3}$ \\ ${ }^{1}$ Department of Mathematics, Acharya Nagarjuna University, \\ Nagarjuna Nagar, Guntur - 522 510, Andhra Pradesh, India \\ ${ }^{2}$ Department of Mathematics, Baba Institute of Technology and Sciences, \\ P.M.Palem, Madhurawada Visakhapatnam - 530048, Andhra Pradesh, India \\ ${ }^{3}$ Department of Mathematics, Ramachandra College of Engineering, \\ Vatluru(V), Eluru-534007, West Godavari Dist., Andhra Pradesh, India \\ *Corresponding Author: kishore.apr2@gmail.com
}

Copyright @2013 Horizon Research Publishing All rights reserved.

\begin{abstract}
In this paper, we have given a unique common fixed point theorem for four mappings in a cone metric type space which is a slight variant of theorems of $[1,10]$. We also gave an example to illustrate our main theorem. Finally we have given an example in which theorem of [10] is not applicable whereas our corollary is applicable.
\end{abstract}

Keywords Cone Metric Type Space, Weakly Compatible Maps, Common Fixed Points

2000 Mathematics Subject Classification: 54H25, 47H10, 54E50

\section{Introduction}

Replacing the real numbers, as the co-domain of a metric, by an ordered Banach space, the authors Huang and Zhang [8] introduced the concept of cone metric spaces. They described the convergence in cone metric spaces, introduced their completeness and proved some fixed point theorems for contractive mappings. Cones and ordered normed spaces have some applications in optimization theory . The initial work of Huang and Zhang [8] inspired many authors to prove fixed point theorems and common fixed point theorems for two or more mappings on cone metric spaces, for example $[2,3,4,5,6,7,11,12,13]$ etc.

First we state some known definitions and lemmas.

Definition 1.1 ([8]) Let $E$ be a real Banach space and $P$ be a subset of $E . P$ is called a cone if and only if :

(i) $P$ is closed, non - empty and $P \neq\{0\}$;

(ii) $a, b \in R, a, b \geq 0, x, y \in P \Rightarrow a x+b y \in P$

(iii) $P \cap(-P)=\{0\}$.

For a given cone $P$, we define a partial ordering $\leq$ with respect to $P$ by $x \leq y$ if and only if $x-y \in P$. We write $x<y$ to indicate that $x \leq y$ but $x \neq y$, while $x<<y$ will stand for $y-x \in \operatorname{Int} P$, Int $P$ denotes the interior of $P$.

The cone $P \subseteq E$ is said to be solid if Int $P \neq \emptyset$.

Analogously with the definition of cone metric spaces in [8], we consider the cone metric type spaces given by $[9]$.

Definition 1.2 ([9]) Let $X$ be a nonempty set and $E$ a real Banach space with cone $P$. A vector valued function $d: X \times X \rightarrow E$ is said to be a cone metric type function on $X$ with constant $k \geq 1$ if the following conditions are satisfied

$\left(d_{1}\right) 0 \leq d(x, y)$ for all $x, y \in X$ and $d(x, y)=0$ if and only if $x=y$;

$\left(d_{2}\right) d(x, y)=d(x, y)$ for all $x, y \in X$

$\left(d_{3}\right) d(x, y) \leq k[d(x, z)+d(z, y)]$ for all $x, y, z \in X$. 
The pair $(X, d)$ is called a cone metric type space (shortly CMTS).

For $k=1$ in Definition 1.2, we obtain a cone metric space introduced in [8].

Definition $1.3([9])$ : Let $(X, d)$ be a CMTS and $\left\{x_{n}\right\}$ a sequence in $X$

(i) $\left\{x_{n}\right\}$ converges to $x \in X$ if for every $c \in E$ with $0<<c$, there exists $n_{0} \in N$ such that $d\left(x_{n}, x\right)<<c$ for all $n>n_{0}$. We write $\lim _{n \rightarrow \infty} x_{n}=x$ or $x_{n} \rightarrow x$ as $n \rightarrow \infty$.

(ii) If for every $c \in E$ with $0<<c$, there exists $n_{0} \in N$ such that $d\left(x_{n}, x_{m}\right)<<c$ for all $n>n_{0}, m>n_{0}$, then $\left\{x_{n}\right\}$ is called a Cauchy sequence in $X$.

(iii) If every Cauchy sequence is convergent in $X$, then $X$ is called a complete CMTS.

The following properties hold in the case of CMTS.

Lemma 1.4 ([9]): Let $(X, d)$ be a CMTS over ordered real Banach space E with a cone P. The following properties hold $(a, b, c \in E)$.

(i) If $a \preceq b$ and $b<<c$ then $a<<c$

(ii) If $0 \leq a<<c$ for all $c \in$ Int $P$, then $a=0$.

(iii) If $a \preceq \lambda a$, where $a \in P$ and $0 \leq \lambda<1$ then $a=0$.

(iv) Let $x_{n} \rightarrow 0$ in $E$ and let $0<<c$. Then there exists $n_{0} \in N$ such that $x_{n}<<c$ for each $n>n_{0}$

Aleksandar et al. [1] proved the following theorem.

Theorem $1.5([1])$ : Let $(X, d)$ be a CMTS with constant $1 \leq k \leq 2$ and $P$ a solid cone. Suppose that $F, G, S, T$ : $X \rightarrow X$ are such that

(i) $S(X) \subseteq G(X), T(X) \subseteq F(X)$,

(ii) for some constant $\lambda \in\left(0, \frac{1}{k}\right)$ for all $x, y \in X$ there exists

$$
u(x, y) \in\left\{k d(F x, G y), k d(F x, S x), k d(G y, T y), k \frac{[d(F x, T y)+d(G y, T y)]}{2}\right\}
$$

such that $d(S x, T y) \leq \frac{\lambda}{k} u(x, y)$ holds

(iii) one of $S(X), T(X), F(X)$ and $G(X)$ is a complete sub space of $X$

(iv) the pairs $(S, F)$ and $(T, G)$ are weakly compatible.

Then $F, G, S$ and $T$ have a unique common fixed point.

Reecently Marija P. Stanic et al [10] proved common fixed point theorem under contractive condition of Ćirić's type on cone metric type spaces.

Theorem 1.6 ([10]): Let $(X, d)$ be a CMTS with constant $k \in[1,2]$ relative to a solid cone $P$. Let $S, T: X \rightarrow X$ be such that for some constant $\lambda \in\left(0, \frac{1}{2 k}\right)$ for all $x, y \in X$ there exists

$$
u(x, y) \in\{d(x, y), d(x, S x), d(y, T y), d(x, T y), d(y, S x)\}
$$

such that $d(S x, T y) \leq \lambda u(x, y)$ holds.

Then $S$ and $T$ have a unique common fixed point.

In this paper we prove a unique common fixed point theorem for four mappings which is a slight variant of Theorem 1.5 [1] and Theorem $1.6[10]$ when $F=G=I$. (Identity map)

\section{Results}

Theorem 2.1 Let $(X, d)$ be a cone metric type space with constant $k \in[1,2]$ relative to a solid cone $P$. Let $S, T, F, G: X \rightarrow X$ be satisfying

(2.1.1) $d(S x, T y) \preceq \lambda u(x, y)$, where

$$
u(x, y) \in\left\{d(F x, G y), d(F x, S x), d(G y, T y), \frac{1}{2 k} d(F x, T y), \frac{1}{2 k} d(G y, S x)\right\}
$$

for all $x, y \in X$, where $0<\lambda<1$ and $\lambda k<1$,

(2.1.2) $S(X) \subseteq G(X), T(X) \subseteq F(X)$,

(2.1.3) one of $F(X), G(X), S(X)$ and $T(X)$ is a complete sub space of $X$,

(2.1.4) the pairs $(S, F)$ and $(T, G)$ are weakly compatible.

Then $S, T, F$ and $G$ have a unique common fixed point in $X$. 
Let $x_{0} \in X$.

Define $y_{2 n}=S x_{2 n}=G x_{2 n+1}$,

$y_{2 n+1}=T x_{2 n+1}=F x_{2 n+2}, n=0,1,2, \cdots$.

$$
\begin{aligned}
d\left(y_{2 n+2}, y_{2 n+1}\right) & =d\left(S x_{2 n+2}, T x_{2 n+1}\right) \\
& \preceq \lambda u\left(x_{2 n+2}, x_{2 n+1}\right),
\end{aligned}
$$

where

$$
u\left(x_{2 n+2}, x_{2 n+1}\right) \in\left\{d\left(y_{2 n+1}, y_{2 n}\right), d\left(y_{2 n+1}, y_{2 n+2}\right), d\left(y_{2 n}, y_{2 n+1}\right), 0, \frac{1}{2 k} d\left(y_{2 n}, y_{2 n+2}\right)\right\}
$$

we have the following cases

- $d\left(y_{2 n+2}, y_{2 n+1}\right) \preceq \lambda d\left(y_{2 n+1}, y_{2 n}\right)$ or

- $d\left(y_{2 n+2}, y_{2 n+1}\right) \preceq \lambda d\left(y_{2 n+1}, y_{2 n+2}\right)$ which implies that $d\left(y_{2 n+1}, y_{2 n+2}\right)=0$ or

- $d\left(y_{2 n+2}, y_{2 n+1}\right) \preceq \frac{\lambda}{2 k} d\left(y_{2 n}, y_{2 n+2}\right) \preceq \frac{\lambda}{2}\left[d\left(y_{2 n}, y_{2 n+1}\right)+d\left(y_{2 n+1}, y_{2 n+2}\right)\right]$.

That is $d\left(y_{2 n+2}, y_{2 n+1}\right) \preceq \frac{\lambda}{2-\lambda} d\left(y_{2 n}, y_{2 n+1}\right) \preceq \lambda d\left(y_{2 n}, y_{2 n+1}\right)$.

In all cases, $d\left(y_{2 n+2}, y_{2 n+1}\right) \preceq \lambda d\left(y_{2 n+1}, y_{2 n}\right)$,

Similarly we can show that $d\left(y_{2 n+1}, y_{2 n}\right) \preceq \lambda d\left(y_{2 n}, y_{2 n-1}\right)$.

Thus $d\left(y_{n+1}, y_{n}\right) \preceq \lambda d\left(y_{n}, y_{n-1}\right)$, so that

$$
d\left(y_{n+1}, y_{n}\right) \preceq \lambda^{n} d\left(y_{0}, y_{1}\right)
$$

For $m>n$, we have

$$
\begin{aligned}
d\left(y_{n}, y_{m}\right) & \preceq k d\left(y_{n}, y_{n+1}\right)+k^{2} d\left(y_{n+1}, y_{n+2}\right)+\cdots+k^{m-n-1} d\left(y_{m-1}, y_{m}\right) \\
& \preceq\left(k \lambda^{n}+k^{2} \lambda^{n+1}+\cdots+k^{m-n-1} \lambda^{m-1}\right) d\left(y_{0}, y_{1}\right) \\
& \preceq \frac{k \lambda^{n}}{1-k \lambda} d\left(y_{0}, y_{1}\right), \text { since } k \lambda<1 .
\end{aligned}
$$

Now by (iv) and (i) of Lemma 1.4, it follows that for every $c \in \operatorname{Int} P$, there exists $n_{0} \in N$ such that $d\left(y_{n}, y_{m}\right)<<c$ for every $m>n>n_{0}$. Thus $\left\{y_{n}\right\}$ is a Cauchy sequence.

Suppose that $F(X)$ is a complete sub space of $X$.

Since $\left\{y_{2 n+1}\right\}=\left\{F x_{2 n+2}\right\} \subseteq F(X)$, there exist $z$ and $p$ in $X$ such that $y_{2 n+1} \rightarrow z=F(p)$. Since $\left\{y_{n}\right\}$ is Cauchy, we have $y_{2 n} \rightarrow z$.

$$
\begin{aligned}
d(S p, F p) & \preceq k\left[d\left(S p, y_{2 n+1}\right)+d\left(y_{2 n+1}, F p\right)\right] \\
& =k d\left(S p, T x_{2 n+1}\right)+k d\left(y_{2 n+1}, z\right) \\
& \preceq k \lambda u\left(p, x_{2 n+1}\right)+k d\left(y_{2 n+1}, z\right),
\end{aligned}
$$

where

$$
u\left(p, x_{2 n+1}\right) \in\left\{d\left(F p, y_{2 n}\right), d(F p, S p), d\left(y_{2 n}, y_{2 n+1}\right), \frac{1}{2 k} d\left(F p, y_{2 n+1}\right), \frac{1}{2 k} d\left(y_{2 n}, S p\right)\right\}
$$

For any $0<<c$ and sufficiently large $n$, at least one of the following cases hold:

- $d(S p, F p) \preceq k \lambda d\left(z, y_{2 n}\right)+k d\left(y_{2 n+1}, z\right)<<k \lambda \frac{c}{2 k \lambda}+k \frac{c}{2 k}=c ;$

- $d(S p, F p) \preceq k \lambda d(F p, S p)+k d\left(y_{2 n+1}, z\right)$

Thus

$$
d(S p, F p) \preceq \frac{k}{1-k \lambda} d\left(y_{2 n+1}, z\right)<<\frac{k}{1-k \lambda} \frac{c(1-k \lambda)}{k}=c ;
$$

$$
\begin{aligned}
d(S p, F p) & \preceq k \lambda d\left(y_{2 n}, y_{2 n+1}\right)+k d\left(y_{2 n+1}, z\right) \\
& \preceq k^{2}\left[d\left(y_{2 n}, z\right)+d\left(z, y_{2 n+1}\right)\right]+k d\left(y_{2 n+1}, z\right) \\
& <\quad \lambda k^{2} \frac{c}{2 \lambda k^{2}}+\left(\lambda k^{2}+k\right) \frac{c}{2\left(\lambda k^{2}+k\right)}=c ;
\end{aligned}
$$

- $d(S p, F p) \preceq \frac{\lambda}{2} d\left(z, y_{2 n+1}\right)+k d\left(y_{2 n+1}, z\right)<<\left(\frac{\lambda}{2}+k\right) \frac{c}{\left(\frac{\lambda}{2}+k\right)}=c ;$

$$
\begin{aligned}
d(S p, F p) & \preceq \frac{\lambda}{2} d\left(y_{2 n}, S p\right)+k d\left(y_{2 n+1}, z\right) \\
& \preceq \frac{\lambda}{2}\left[d\left(y_{2 n}, z\right)+d(F p, S p)\right]+k d\left(y_{2 n+1}, z\right)
\end{aligned}
$$


i.e.

$$
\begin{aligned}
d(S p, F p) & \preceq \frac{\lambda \frac{k}{2}}{\left(1-\lambda \frac{k}{2}\right)} d\left(y_{2 n}, z\right)+\frac{k}{\left(1-\lambda \frac{k}{2}\right)} d\left(y_{2 n+1}, z\right) \\
& <<\frac{\lambda k}{(2-\lambda k)} \frac{(2-\lambda k)}{\lambda k} \frac{c}{2}+\frac{2 k}{(2-\lambda k)} \frac{(2-\lambda k)}{k} \frac{c}{4}=c .
\end{aligned}
$$

Thus in all cases, we have

$$
d(S p, F p)<<c \text { for every } c \in \operatorname{Int} P \text {. }
$$

Hence $S p=F p=z$.

Since the pair $(F, S)$ is weakly compatible, we have $F z=S z$.

$$
\begin{aligned}
d(S z, z) & \preceq k d\left(S z, y_{2 n+1}\right)+k d\left(y_{2 n+1}, z\right) \\
& =k d\left(S z, T x_{2 n+1}\right)+k d\left(y_{2 n+1}, z\right) \\
& \preceq k \lambda u\left(z, x_{2 n+1}\right)+k d\left(y_{2 n+1}, z\right),
\end{aligned}
$$

where

$$
\begin{aligned}
u\left(z, x_{2 n+1}\right) & \in\left\{d\left(F z, y_{2 n}\right), 0, d\left(y_{2 n}, y_{2 n+1}\right), \frac{1}{2 k} d\left(F z, y_{2 n+1}\right), \frac{1}{2 k} d\left(y_{2 n}, S z\right)\right\} \\
& =\left\{d\left(S z, y_{2 n}\right), 0, d\left(y_{2 n}, y_{2 n+1}\right), \frac{1}{2 k} d\left(S z, y_{2 n+1}\right), \frac{1}{2 k} d\left(y_{2 n}, S z\right)\right\} .
\end{aligned}
$$

We have the following cases:

- $d(S z, z) \preceq k \lambda d\left(S z, y_{2 n}\right)+k d\left(y_{2 n+1}, z\right)$

$$
\preceq \lambda k^{2} d(S z, z)+\lambda k^{2} d\left(z, y_{2 n}\right)+k d\left(y_{2 n+1}, z\right)
$$

$$
\text { i. e. } \begin{aligned}
d(S z, z) \preceq & \frac{\lambda k^{2}}{\left(1-\lambda k^{2}\right)} d\left(y_{2 n}, z\right)+\frac{k}{\left(1-\lambda k^{2}\right)} d\left(y_{2 n+1}, z\right) \\
& <<\frac{\lambda k^{2}}{\left(1-\lambda k^{2}\right)} \frac{\left(1-\lambda k^{2}\right)}{\lambda k^{2}} \frac{c}{2}+\frac{k}{\left(1-\lambda k^{2}\right)} \frac{\left(1-\lambda k^{2}\right)}{k} \frac{c}{2}=c ;
\end{aligned}
$$

- $d(S z, z) \preceq k \lambda d\left(y_{2 n}, y_{2 n+1}\right)+k d\left(y_{2 n+1}, z\right)$

$$
\begin{aligned}
& \preceq \lambda k^{2} d\left(y_{2 n}, z\right)+\lambda k^{2} d\left(z, y_{2 n+1}\right)+k d\left(y_{2 n+1}, z\right) \\
& <<\lambda k^{2} \frac{c}{2 \lambda k^{2}}+\left(\lambda k^{2}+k\right) \frac{c}{2\left(\lambda k^{2}+k\right)}=c ;
\end{aligned}
$$

- $d(S z, z) \preceq \frac{\lambda}{2} d\left(S z, y_{2 n+1}\right)+k d\left(y_{2 n+1}, z\right)$

$$
\begin{aligned}
\preceq \frac{\lambda k}{2} d(S z, z) & +\frac{\lambda k}{2} d\left(z, y_{2 n+1}\right)+k d\left(y_{2 n+1}, z\right) \\
\text { i. e. } d(S z, z) \preceq & \frac{\left(\frac{\lambda k}{2}+k\right)}{\left(1-\frac{\lambda k}{2}\right)} d\left(z, y_{2 n+1}\right) \\
& <<\frac{\left(\frac{\lambda k}{2}+k\right)}{\left(1-\frac{\lambda k}{2}\right)} \frac{\left(1-\frac{\lambda k}{2}\right)}{\left(\frac{\lambda k}{2}+k\right)} c=c ;
\end{aligned}
$$

- $d(S z, z) \preceq \frac{\lambda}{2} d\left(y_{2 n}, S z\right)+k\left(y_{2 n+1}, z\right)$

$$
\begin{aligned}
\preceq & \frac{\lambda}{2} k d\left(y_{2 n}, z\right)+\frac{\lambda}{2} k d(z, S z)+k d\left(y_{2 n+1}, z\right) \\
\text { i.e. } d(S z, z) \preceq & \left(\frac{\lambda \frac{k}{2}}{1-\lambda \frac{k}{2}}\right) d\left(y_{2 n}, z\right)+\frac{k}{\left(1-\lambda \frac{k}{2}\right)} d\left(y_{2 n+1}, z\right) \\
& <<\frac{\lambda k}{(2-\lambda k)} \frac{(2-\lambda k)}{\lambda k} \frac{c}{2}+\frac{2 k}{(2-\lambda k)} \frac{(2-\lambda k)}{k} \frac{c}{4}=c .
\end{aligned}
$$

Thus in all cases, we have

$$
d(S z, z)<<c \text { for every } c \in \operatorname{Int} P .
$$

Hence $S z=z$. Thus $F z=S z=z$.

Since $S(X) \subseteq G(X)$, there exists $v \in X$ such that $z=S z=G v$. $d(z, T v)=d(S z, T v) \preceq \lambda u(z, v)$, where

$$
u(z, v) \in\left\{0,0, d(z, T v), \frac{1}{2 k} d(z, T v), 0\right\} .
$$


Since $1 \leq k \leq 2$ and $\lambda<1$, it follows that $T v=z$.

Thus $G v=z=T v$.

Since the pair $(T, G)$ is a weakly compatible, we have $G z=T z$.

$d(z, T z)=d(S z, T z) \preceq \lambda u(z, z)$, where

$$
u(z, z) \in\left\{d(z, T z), 0,0, \frac{1}{2 k} d(z, T z), \frac{1}{2 k} d(z, T z)\right\} .
$$

Since $1 \leq k \leq 2$ and $\lambda<1$, it follows that $T z=z$.

Thus $G z=T z=z$.

Hence $z$ is a common fixed point of $S, T, F$ and $G$.

Uniqueness of common fixed point follows easily from (2.1.1).

Now we give an example which illustrates our main Theorem 2.1

Example 2.2 Let $X=[0, \infty), E=R$ and $P=[0, \infty)$. Define $d(x, y)=|x-y|^{2}, \forall x, y \in X$, then

$$
\begin{aligned}
d(x, y) & =|x-y|^{2} \\
& \leq[|x-z|+|z-y|]^{2} \\
& =|x-z|^{2}+|z-y|^{2}+2|x-z||z-y| \\
& \leq 2\left[|x-z|^{2}+|z-y|^{2}\right] \\
& =2[d(x, z)+d(z, y)]
\end{aligned}
$$

Thus $(X, d)$ is not a cone metric space and it is a cone metric type space with constant $k=2$.

Let $S, T, F$ and $G$ be self maps on $X$ defined by

$S(x)=\frac{1}{\sqrt{3}} \sin 2 x, T(x)=\frac{1}{\sqrt{3}} \sin 3 x, F(x)=2 x$ and $G(x)=3 x$.

Then

$$
\begin{aligned}
d(S x, T y) & =\left|\frac{1}{\sqrt{3}} \sin 2 x-\frac{1}{\sqrt{3}} \sin 3 y\right|^{2} \\
& =\frac{1}{3}\left|2 \cos \left(\frac{2 x+3 y}{2}\right) \sin \left(\frac{2 x-3 y}{2}\right)\right|^{2} \\
& \leq \frac{1}{3}|2 x-3 y|^{2} \\
& =\frac{1}{3} d(F x, G y) .
\end{aligned}
$$

Clearly (2.1.2), (2.1.3) and (2.1.4) are satisfied and 0 is the unique common fixed point of $S, G, F$ and $G$.

Corollary 2.3 Let $(X, d)$ be a cone metric type space with constant $k \in[1,2]$ relative to a solid cone P. Let $S, T: X \rightarrow X$ be satisfying $d(S x, T y) \preceq \lambda u(x, y)$, where

$$
u(x, y) \in\left\{d(x, y), d(x, S x), d(y, T y), \frac{1}{2 k} d(x, T y), \frac{1}{2 k} d(y, S x)\right\}
$$

for all $x, y \in X$, where $0<\lambda<1$ and $k \lambda<1$.

Then $S$ and $T$ have a unique common fixed point in $X$.

Now we give an example in which Corollary 2.3 is applicable whereas Theorem 1.6 of [10] is not applicable.

Example 2.4 Let $(X, d), E$ and $P$ be as in Example 2.2

Let $S x=\frac{1}{\sqrt{3}} \sin x \forall x \in X$

Then

$$
\begin{aligned}
d(S x, S y) & =\left|\frac{1}{\sqrt{3}} \sin x-\frac{1}{\sqrt{3}} \sin y\right|^{2} \\
& =\frac{1}{3}\left|2 \cos \left(\frac{x+y}{2}\right) \sin \left(\frac{x-y}{2}\right)\right|^{2} \\
& \leq \frac{1}{3}|x-y|^{2} \\
& =\frac{1}{3} d(x, y)
\end{aligned}
$$

Here $\lambda=\frac{1}{3} \notin\left(0, \frac{1}{4}\right)=\left(0, \frac{1}{2 k}\right)$.

Hence Theorem 1.6 [10] is not applicable with $T=S$.

Clearly Corollary 2.3 is applicable with $T=S$. 


\section{Discussion and Conclusion}

Our main Theorem 2.1 is a slight variant of Theorem 1.5. We have given an Example 2.2 to illustate our main Theorem 2.1. We also show that our Corollary 2.3 is applicable whereas Theorem 1.6 is not applicable by means Example 2.4

\section{REFERENCES}

[1] Aleksandar S. Cvetković, Marija P. Stanić, Sladjana Dimitrijević and Suzana Simić, Common Fixed Point Theorems for Four Mappings on Cone Metric Type Space, Fixed Point Theory and Applications, Volume 2011, Article ID 589725, 15 pages, doi:10.1155/2011/589725.

[2] D. Turkoglu, M. Abuloha and T. Abdeljawad, KKM mappings in cone metric spaces and some fixed point theorems, Nonlinear Analysis: Theory, Methods and Applications, 72(1), (2010), 348 - 353.

[3] D. Turkoglu, M. Abuloha and T. Abdeljawad, Fixed points of generalized contraction mappings in cone metric spaces, Mathematical Communications, 16, (2011), 325 - 334.

[4] E. Karapínar, Fixed point theorems in cone Banach spaces, Fixed Point Theory Appl., Volume 2009, (2009), 9 pages, Article ID 609281.

[5] E. Karapínar, Some non - unique fixed point theorems of Ciric type on cone metric spaces, Abstr. Appl. Anal., Volume 2010, (2010), 14 pages, Article ID 123094, doi:10.1155/2010/123094.

[6] E. Karapínar and D. A. Turkoglu, Best approximations theorem for a couple in cone Banach Space, Fixed Point Theory Appl., Volume 2010, (2010), 9 pages , Article ID 784578.

[7] I. Altun, B. Damjanovic and D. Djoric, Fixed point and common fixed point theorems on ordered cone metric spaces, Applied Mathematics Letters, 23(3), (2010), $310-316$.

[8] L. G. Huang and X. Zhang, Cone metric spaces and fixed point theorems of Contractive mappings, J. Math.Anal.Appl.,332(2), (2007), 1468 - 1476.

[9] M. A. Khamsi and N. Hussain, KKM mappings in metric type spaces, Nonlinear Analysis : Theory, Methods and Applications, 73(9), (2010), 3123 - 3129.

[10] Marija P. Stanic, Aleksandar S. Cvetkovic, Suzana Simic, Sladjana Dimitrijevic, Common fixed point under contractive condition of Ciric's type on cone metric type spaces, Fixed Point Theory and Applications 2012, 2012:35 doi:10.1186/1687-1812-2012-35 .

[11] Sh. Rezapour, R. Hamlbarani, Some Notes on the Paper "Cone Metric Spaces and Fixed Point Theorems of Contractive Mappings", J. Math. Anal. Appl., 347(2008), 719 - 724.

[12] T. Abdeljawad and E. Karapínar, Quasi - cone metric spaces and generalizations of Caristi Kirk's theorem, Fixed Point Theory Appl., Volume 2009 (2009), Article ID 574387, 9 pages.

[13] T. Abdeljawad, E. Karapínar and K.Tas, Common fixed point theorems in cone Banach spaces, Hacet.J.Math.Stat., 40(2), (2011), $211-217$. 